\title{
Global existence of positive periodic solutions of a general differential equation with neutral type
}

\author{
Ming Liu', Jun $\mathrm{CaO}^{1 *}$ (i) and Xiaofeng $\mathrm{Xu}^{2}$
}

"Correspondence:

juncao_nefu@163.com

${ }^{1}$ Northeast Forestry University,

Harbin, Heilongjiang 150040,

P.R. China

Full list of author information is

available at the end of the article

\begin{abstract}
In this paper, the dynamics of a general differential equation with neutral type are investigated. Under certain assumptions, the stability of positive equilibrium and the existence of Hopf bifurcation are obtained by analyzing the distribution of eigenvalues. And global existence of positive periodic solutions is established by using the global Hopf bifurcation result of Krawcewicz et al. Finally, by taking neutral Nicholson's blowflies model and neutral Mackey-Glass model as two examples, some numerical simulations are carried out to illustrate the analytical results.
\end{abstract}

Keywords: Differential equation; Neutral; Stability; Global Hopf bifurcation

\section{Introduction}

The standard age-structure model is as follows:

$$
\begin{aligned}
& u_{t}+u_{a}=-\mu(a, w) u, \\
& u(t, 0)=\int_{0}^{\infty} b(a, w) u(t, a) d a, \\
& w(t)=\int_{0}^{\infty} \rho(a) u(t, a) d a,
\end{aligned}
$$

with the initial condition $u(0, a)=u_{0}(a)$. Here, $u(t, a)$ is the age distribution at time $t$, which satisfies $u(t, \tau)=u(0, \tau-t)$ and $u(t, \infty)=0$. Function $w$ is a weighted average of the total population with weight function $\rho(a) \geq 0$. Functions $b$ and $\mu$ denote the birth and death rates, respectively, which all depend on the age and the average $w$.

Let $\tau$ be the critical age that separates adults and juveniles. Then the total population of the mature individuals is

$$
N(t)=\int_{\tau}^{\infty} u(t, a) d a .
$$

(c) The Author(s) 2021. This article is licensed under a Creative Commons Attribution 4.0 International License, which permits use, sharing, adaptation, distribution and reproduction in any medium or format, as long as you give appropriate credit to the original author(s) and the source, provide a link to the Creative Commons licence, and indicate if changes were made. The images or other third party material in this article are included in the article's Creative Commons licence, unless indicated otherwise in a credit line to the material. If material is not included in the article's Creative Commons licence and your intended use is not permitted by statutory regulation or exceeds the permitted use, you will need to obtain permission directly from the copyright holder. To view a copy of this licence, visit http://creativecommons.org/licenses/by/4.0/ 
Assume that the death rate $\mu$ is a constant step function

$$
\mu(a, w)=r H_{\tau}(a)
$$

where $H$ is the Heaviside function with jump at $a=\tau$ and

$$
\rho(a)=H_{\tau}(a)
$$

which implies $w(t)=N(t)$ and the birth rate only depends on the age $a$ and the population of the mature individuals $N$. Further assume that the juveniles do not reproduce and the birth rate $b$ has a delta peak at $a=\tau$

$$
b(a, N)=g(N) H_{\tau}(a)+c \delta_{\tau}(a),
$$

where $\delta$ is the delta function. Then it can be verified that $N(t)$ satisfies

$$
\dot{N}(t)-c \dot{N}(t-\tau)=-r N(t)+c r N(t-\tau)+f(N(t-\tau)),
$$

where $f(s)=s g(s)$. For more details on the derivation of Eq. (1), we refer the reader to [1].

Model (1) is a neutral differential equation, and the corresponding delay equation $(c=0)$ describes many famous models for population growth, which include Nicholson's blowflies model

$$
\dot{N}(t)=-r N(t)+p N(t-\tau) e^{-\alpha N(t-\tau)}
$$

the Mackey-Glass models

$$
\begin{aligned}
& \dot{N}(t)=-r N(t)+\frac{b N(t-\tau)}{1+N^{m}(t-\tau)}, \\
& \dot{N}(t)=-r N(t)+\frac{b}{1+N^{m}(t-\tau)},
\end{aligned}
$$

and the Lasota-Wazewska model

$$
\dot{N}(t)=-r N(t)+p e^{-\alpha N(t-\tau)} .
$$

Nicholson's blowflies model (2) has been extensively studied in the literature, where its results mainly concern the global attractivity of positive equilibrium and oscillatory behaviors of solutions (see [2-6]). Several studies have also been carried out on model (2) with time periodic coefficients (see [7, 8]), on discrete Nicholson's blowflies model (see [9-12]), and on diffusive Nicholson's blowflies model (see [13-17]). Mackey-Glass equations (3) and (4) and Lasota-Wazewska model (5) have been studied in [18-23]. A majority of results deal with the global attractivity of the positive equilibrium and the Hopf bifurcation problem. Meanwhile, there are several papers on the complex dynamics of model (3). For example, Mackey and Glass [24] and Namajūnas et al. [25] studied the chaotic behavior, while Losson et al. [26] investigated the multistability. (Further, we refer to [27] 
and [28] for biological models, involving chemotaxis and nonlinear diffusive mechanism, formulated by the introduction of reactions coupling growth and death impacts.)

Wei investigated the global Hopf bifurcation of Eq. (1) with $c=0$ [29], and Li et al. investigated the global Hopf bifurcation of Eq. (1) with $f(s)=p s e^{-\alpha s}$ [30]. The purpose of the present paper is to study the global Hopf bifurcation of the general neutral differential equation (1). Here, $r>0,0<c<1$, and $f$ is a nonnegative and $C^{2}$ function in $[0, \infty)$ with $f(\infty)=0$ and satisfies one of the following assumptions:

(H1) $f(0)>0$ and $f(s)$ is strictly monotone decreasing when $s \in(0, \infty)$.

(H2) $f(0)=0$, and $\frac{f(s)}{s}$ is strictly monotone decreasing when $s \in(0, \infty)$.

It is easy to see that the function $f$ in (2) or (3) satisfies (H2) and the function $f$ in (4) or (5) satisfies (H1).

The rest of the paper is organized as follows. In Sect. 2, we investigate the existence of positive equilibrium and analyze the distribution of the roots of the characteristic equation to give various conditions on the stability of positive equilibrium and the existence of Hopf bifurcation. In Sect. 3, we establish the extended existence of bifurcation periodic solutions by using the global Hopf bifurcation result of Krawcewicz et al. [31]. In Sect. 4, we carry out some numerical simulations to support the analytical results.

\section{Stability and local Hopf bifurcation analysis}

In this section, we consider the stability of positive equilibrium and the existence of local Hopf bifurcation by taking time delay $\tau$ as a bifurcation parameter.

For neutral differential equations, positive initial conditions can lead to positive solutions is not a trivial matter. Here, we provide some positively invariant sets for Eq. (1). Without loss of generality, assume that the initial time for Eq. (1) is zero and define

$$
\begin{aligned}
\Gamma_{1}= & \{\phi \in C([-\tau, 0], \mathbb{R}) \mid \phi(0)-c \phi(-\tau) \geq 0, \text { and } \phi(\theta) \geq 0, \theta \in[-\tau, 0]\}, \\
\Gamma_{2}= & \left\{\phi \in C([-\tau, 0], \mathbb{R}) \mid \text { the left derivative } D^{-} \phi(\theta) \text { exists, } D^{-} \phi(\theta)\right. \\
& +r \phi(\theta) \geq 0, \text { and } \phi(\theta) \geq 0, \theta \in(-\tau, 0]\} .
\end{aligned}
$$

Then we have the following result.

Theorem 2.1 If $\Gamma=\Gamma_{1}$ or $\Gamma=\Gamma_{2}$, then $\Gamma$ is a positively invariant set for Eq. (1).

Proof Assume that $\theta \in[-\tau, 0]$ and $\phi \in \Gamma$. Then, for $t \in(0, \tau]$, we have $N(t-\tau)=\phi(t-\tau) \geq$ 0 . Since function $f$ is nonnegative in $[0, \infty)$, we can obtain

$$
\begin{aligned}
& \quad \dot{N}(t)-c \dot{N}(t-\tau) \geq-r(N(t)-c N(t-\tau)) . \\
& \text { If } \Gamma=\Gamma_{1} \text {, one has } \\
& \qquad N(t)-c N(t-\tau) \geq(N(0)-c N(-\tau)) e^{-r t}=(\phi(0)-c \phi(-\tau)) e^{-r t} \geq 0,
\end{aligned}
$$

and

$$
N(t) \geq c N(t-\tau)=c \phi(t-\tau) \geq 0 .
$$

Therefore, by mathematical induction, $\Gamma_{1}$ is a positively invariant set for Eq. (1). 
If $\Gamma=\Gamma_{2}$, then the solution $N(t)$ of Eq. (1) satisfies that $D^{-} N(t)$ exists,

$$
D^{-} N(t)+r N(t) \geq c\left(D^{-} N(t-\tau)+r N(t-\tau)\right)=c\left(D^{-} \phi(t-\tau)+r \phi(t-\tau)\right) \geq 0,
$$

and

$$
N(t) \geq N(0) e^{-r t}=\phi(0) e^{-r t} \geq 0 .
$$

Therefore, by mathematical induction, $\Gamma_{2}$ is a positively invariant set for Eq. (1).

In the remainder of the paper, we investigate the dynamics of Eq. (1) in a positively invariant region $\Gamma$ with either $\Gamma=\Gamma_{1}$ or $\Gamma=\Gamma_{2}$, and formulate our results accordingly.

Theorem 2.2 Assume that $r>0,0<c<1$, and $f$ is a nonnegative and $C^{2}$ function in $[0, \infty)$ with $f(\infty)=0$.

(i) If (H1) holds, then Eq. (1) has a unique positive equilibrium $N_{0}$.

(ii) If $(\mathrm{H} 2)$ and $f^{\prime}(0)>r(1-c)$ hold, then Eq. (1) has a unique positive equilibrium $N_{0}$.

Proof Obviously, the positive equilibrium $N_{0}$ of Eq. (1) satisfies

$$
g\left(N_{0}\right):=-r(1-c) N_{0}+f\left(N_{0}\right)=0 .
$$

If (H1) holds, then we have $g(0)=f(0)>0, g(\infty)=-\infty$. Since $g^{\prime}(s)=-r(1-c)+f^{\prime}(s)<0$, there exists a unique $N_{0}$ in the interval $(0, \infty)$ such that $g\left(N_{0}\right)=0$. If (H2) holds, we have $\lim _{s \rightarrow 0^{+}} \frac{f(s)}{s}=f^{\prime}(0)>r(1-c)$ and $\lim _{s \rightarrow \infty} \frac{f(s)}{s}=0$. There exists a unique value of $N_{0}$ in the interval $(0, \infty)$ satisfying $\frac{f\left(N_{0}\right)}{N_{0}}=r(1-c)$ due to the monotonicity of $\frac{f(s)}{s}$, which implies that $g(s)=s\left(-r(1-c)+\frac{f(s)}{s}\right)$ has a unique zero $N_{0}$ in the interval $(0, \infty)$. The proof is completed.

Remark 2.3 From the proof of Theorem 2.2, one has that under assumption (H2), if $f^{\prime}(0)>$ $r(1-c)$ does not hold, Eq. (1) has no positive equilibrium. Therefore, in the remainder of this paper, we replace assumption $(\mathrm{H} 2)$ by

$(\mathrm{H} 2)^{\prime} f(0)=0, f^{\prime}(0)>r(1-c)$, and $\frac{f(s)}{s}$ is strictly monotone decreasing when $s \in(0, \infty)$.

The linearization of Eq. (1) at $N=N_{0}$ is given by

$$
\dot{N}(t)-c \dot{N}(t-\tau)=-r N(t)+\left(c r+f^{\prime}\left(N_{0}\right)\right) N(t-\tau) .
$$

Hence, the corresponding characteristic equation is

$$
\Delta(\lambda):=\lambda+r-\left(c r+f^{\prime}\left(N_{0}\right)+c \lambda\right) e^{-\lambda \tau}=0 .
$$

Lemma 2.4 Assume that either (H1) or (H2)' holds. When $\tau=0$, the root of Eq. (6) is negative.

Proof When $\tau=0$, the root of Eq. (6) is

$$
\lambda=\frac{f^{\prime}\left(N_{0}\right)-r(1-c)}{1-c} .
$$


Clearly, we have $f^{\prime}\left(N_{0}\right)<0$ under assumption (H1). Assume that $(\mathrm{H} 2)^{\prime}$ holds. From the monotonicity of $\frac{f(s)}{s}$, we can obtain that

$$
N_{0} f^{\prime}\left(N_{0}\right)-f\left(N_{0}\right)<0
$$

It follows that

$$
f^{\prime}\left(N_{0}\right)<\frac{f\left(N_{0}\right)}{N_{0}}=r(1-c) .
$$

The proof is completed.

Lemma 2.4 implies that the positive equilibrium $N_{0}$ of Eq. (1) is asymptotically stable when $\tau=0$. Thus, with the increase of $\tau$, a stability change at $N_{0}$ can only happen when there are characteristic roots crossing the imaginary axis to the right. In addition, from the proof of Lemma 2.4, one can see that $f^{\prime}\left(N_{0}\right)<r(1-c)$ under assumption $(\mathrm{H} 1)$ or $(\mathrm{H} 2)^{\prime}$. This leads to $\lambda=0$ is not a root of Eq. (6) under assumption (H1) or (H2)'.

Now, let $\lambda=i \omega_{0}\left(\omega_{0}>0\right)$ be a root of Eq. (6). Substituting it into Eq. (6) and separating the real and imaginary parts, we have

$$
\begin{aligned}
& r=\left(c r+f^{\prime}\left(N_{0}\right)\right) \cos \omega_{0} \tau+c \omega_{0} \sin \omega_{0} \tau, \\
& \omega_{0}=-\left(c r+f^{\prime}\left(N_{0}\right)\right) \sin \omega_{0} \tau+c \omega_{0} \cos \omega_{0} \tau .
\end{aligned}
$$

Hence,

$$
\omega_{0}^{2}=\frac{\left(c r+f^{\prime}\left(N_{0}\right)\right)^{2}-r^{2}}{1-c^{2}} .
$$

Since we have $f^{\prime}\left(N_{0}\right)<r(1-c)$ under assumption $(\mathrm{H} 1)$ or $(\mathrm{H} 2)^{\prime}, \omega_{0}>0$ makes sense if and only if

$$
f^{\prime}\left(N_{0}\right)<-r(1+c)
$$

Furthermore, from Eq. (7), one can obtain that

$$
\sin \omega_{0} \tau=\frac{-f^{\prime}\left(N_{0}\right) \omega_{0}}{r^{2}+\omega_{0}^{2}}>0
$$

Therefore, when $f^{\prime}\left(N_{0}\right)<-r(1+c)$, Eq. (6) has a pair of imaginary roots $\pm i \omega_{0}$ if and only if $\tau=\tau_{j}$, where

$$
\tau_{j}=\frac{1}{\omega_{0}}\left(\arccos \frac{r\left(c r+f^{\prime}\left(N_{0}\right)\right)+c \omega_{0}^{2}}{r^{2}+\omega_{0}^{2}}+2 j \pi\right), \quad j=0,1,2, \ldots
$$

From the discussions above, we have the following result.

Lemma 2.5 Assume that either (H1) or (H2)' holds.

(i) If $f^{\prime}\left(N_{0}\right) \geq-r(1+c)$, then Eq. (6) has no imaginary root for any $\tau>0$. 
(ii) If $f^{\prime}\left(N_{0}\right)<-r(1+c)$, then Eq. (6) has a pair of imaginary roots $\pm i \omega_{0}$ when $\tau=\tau_{j}$, where $\tau_{j}$ is defined by Eq. (9).

Lemma 2.6 Assume that either $(\mathrm{H} 1)$ or $(\mathrm{H} 2)^{\prime}$ holds and $f^{\prime}\left(N_{0}\right)<-r(1+c)$ is satisfied. Let $\lambda(\tau)=\alpha(\tau)+i \beta(\tau)$ be the root of Eq. (6) near $\tau=\tau_{j}$ satisfying $\alpha\left(\tau_{j}\right)=0$ and $\beta\left(\tau_{j}\right)=\omega_{0}$. Then we have

$$
\left.\frac{d \alpha}{d \tau}\right|_{\tau=\tau_{j}}>0
$$

Proof Differentiating Eq. (6) with respect to $\lambda$, we have

$$
\frac{d \tau}{d \lambda}=\frac{-f^{\prime}\left(N_{0}\right)}{\lambda(\lambda+r)\left(c r+f^{\prime}\left(N_{0}\right)+c \lambda\right)}-\frac{\tau}{\lambda} .
$$

Thus,

$$
\left.\frac{d \alpha}{d \tau}\right|_{\tau=\tau_{j}}=\frac{\left(r\left(c r+f^{\prime}\left(N_{0}\right)\right)-c \omega_{0}^{2}\right)^{2}+\left(2 c r+f^{\prime}\left(N_{0}\right)\right)^{2} \omega_{0}^{2}}{f^{\prime}\left(N_{0}\right)\left(2 c r+f^{\prime}\left(N_{0}\right)\right)}>0 .
$$

The proof is completed.

By applying Lemmas $2.4,2.5$, and 2.6, one can easily obtain the following stability properties of the positive steady state $N_{0}$ of Eq. (1).

Theorem 2.7 Assume that $r>0,0<c<1, f$ is a nonnegative and $C^{2}$ function in $[0, \infty)$ with $f(\infty)=0$ and either $(\mathrm{H} 1)$ or $(\mathrm{H} 2)^{\prime}$ holds.

(i) If $f^{\prime}\left(N_{0}\right) \geq-r(1+c)$, then for Eq. (1), $N=N_{0}$ is locally asymptotically stable for all $\tau \geq 0$.

(ii) If $f^{\prime}\left(N_{0}\right)<-r(1+c)$, then for Eq. (1), $N=N_{0}$ is locally asymptotically stable for $\tau \in\left[0, \tau_{0}\right)$ and unstable for $\tau>\tau_{0}$. Furthermore, system (1) undergoes a Hopf bifurcation at $N=N_{0}$ when $\tau=\tau_{j}, j=0,1,2, \ldots$.

\section{Global Hopf bifurcation analysis}

In this section, we investigate the global existence of positive periodic solutions of Eq. (1) by using the global Hopf bifurcation theorem [31].

Lemma 3.1 Assume that either (H1) or (H2)' holds. Then all periodic solutions of Eq. (1) are uniformly bounded.

Proof By Theorem 2.1, we know that all periodic solutions of Eq. (1) in $\Gamma$ are bounded below by 0 . Let $u(t)$ be a nonconstant periodic solution of Eq. (1). Then there exist $T^{0}$ and $T_{0}$ such that

$$
\begin{aligned}
& u\left(T^{0}\right)-c u\left(T^{0}-\tau\right)=\max _{t \in \mathbb{R}}(u(t)-c u(t-\tau)), \\
& u\left(T_{0}\right)-c u\left(T_{0}-\tau\right)=\min _{t \in \mathbb{R}}(u(t)-c u(t-\tau)) .
\end{aligned}
$$


Then, for any fixed $t$, we get

$$
u(t) \leq c u(t-\tau)+u\left(T^{0}\right)-c u\left(T^{0}-\tau\right)
$$

Replacing $t$ with $t-\tau$ in the above inequality, we have

$$
u(t-\tau) \leq c u(t-2 \tau)+u\left(T^{0}\right)-c u\left(T^{0}-\tau\right) .
$$

Hence, for any integer $m$,

$$
u(t) \leq c^{m} u(t-m \tau)+\frac{1-c^{m}}{1-c}\left(u\left(T^{0}\right)-c u\left(T^{0}-\tau\right)\right) .
$$

Let $m \rightarrow \infty$ and we can obtain that

$$
u(t) \leq \frac{u\left(T^{0}\right)-c u\left(T^{0}-\tau\right)}{1-c}
$$

By using a similar method, one also has

$$
u(t) \geq \frac{u\left(T_{0}\right)-c u\left(T_{0}-\tau\right)}{1-c}
$$

In addition, from the definitions of $T^{0}$ and $T_{0}$, we know that

$$
\begin{aligned}
& u\left(T^{0}\right)-c u\left(T^{0}-\tau\right)=\frac{1}{r} f\left(u\left(T^{0}-\tau\right)\right), \\
& u\left(T_{0}\right)-c u\left(T_{0}-\tau\right)=\frac{1}{r} f\left(u\left(T_{0}-\tau\right)\right) .
\end{aligned}
$$

Now we can distinguish two cases.

Case (i): (H1) holds.

From Eq. (11), Eq. (12), and Eq. (13), it is easy to see that

$$
u(t) \leq \frac{f\left(u\left(T^{0}-\tau\right)\right)}{r(1-c)} \leq \frac{f(0)}{r(1-c)},
$$

and

$$
u(t) \geq \frac{f\left(u\left(T_{0}-\tau\right)\right)}{r(1-c)} \geq \frac{1}{r(1-c)} f\left(\frac{f(0)}{r(1-c)}\right) .
$$

Case (ii): (H2)' holds.

From $f(0)=f(\infty)=0$ and the continuity of function $f$, we can define

$$
f_{M}=\max _{s \in[0, \infty)} f(s)
$$

Then, by applying Eq. (11) and Eq. (13), we have

$$
u(t) \leq \frac{f\left(u\left(T^{0}-\tau\right)\right)}{r(1-c)} \leq \frac{f_{M}}{r(1-c)}
$$


In particular, from Eq. (12), we have

$$
u\left(T_{0}\right) \geq \frac{u\left(T_{0}\right)-c u\left(T_{0}-\tau\right)}{1-c} .
$$

It follows that

$$
u\left(T_{0}\right) \leq u\left(T_{0}-\tau\right)
$$

Thus, combining with Eq. (13), we can obtain that

$$
-r(1-c) u\left(T_{0}-\tau\right)+f\left(u\left(T_{0}-\tau\right)\right) \leq 0 .
$$

Now, we need to show that $u\left(T_{0}-\tau\right)>0$. In fact, if $u\left(T_{0}-\tau\right)=0$, we can get $u\left(T_{0}\right)=0$ and

$$
u(t)-c u(t-\tau) \geq u\left(T_{0}\right)-c u\left(T_{0}-\tau\right)=0 .
$$

On the other hand, since $u(t)$ is a nonconstant periodic solution of Eq. (1), we have $f(u(t-$ $\tau)) \not \equiv 0$. This leads to

$$
u(t)-c u(t-\tau) \not \equiv 0
$$

Without loss of generality, assume that there exists $t_{*}>0$ such that $u\left(t_{*}\right)-c u\left(t_{*}-\tau\right)>0$. Then we can obtain that, for $t \geq t_{*}$,

$$
u(t)-c u(t-\tau) \geq\left(u\left(t_{*}\right)-c u\left(t_{*}-\tau\right)\right) e^{-r t}>0 .
$$

From the periodicity of $u(t)$, we have $u(t)>c u(t-\tau) \geq 0$ for any $t \in[0, \infty)$, which implies that all nonconstant periodic solutions of Eq. (1) are positive. Hence, we get $u\left(T_{0}-\tau\right)>0$. Then, from Eq. (16), one has

$$
\frac{f\left(u\left(T_{0}-\tau\right)\right)}{u\left(T_{0}-\tau\right)} \leq r(1-c) .
$$

By using the fact that $\frac{f(s)}{s}$ is strictly monotone decreasing when $s \in(0, \infty)$, we can obtain

$$
u\left(T_{0}-\tau\right) \geq N_{0} .
$$

Note that from Eq. (15)

$$
u\left(T_{0}-\tau\right) \leq \frac{f_{M}}{r(1-c)}
$$

Therefore, we can define

$$
f_{m}=\min _{s \in\left[N_{0}, \frac{f_{M}}{r(1-c)}\right]} f(s),
$$


such that

$$
u(t) \geq \frac{f\left(u\left(T_{0}-\tau\right)\right)}{r(1-c)} \geq \frac{f_{m}}{r(1-c)} .
$$

Clearly, $f_{m}$ only depends on function $f$ and parameters $r$ and $c$. Moreover, we have $f_{m}>0$, because $\frac{f(s)}{s}$ is strictly monotone decreasing and tends to zero as $s$ tends to infinity. The proof is completed.

Denote $G=\left[G_{1}, G_{2}\right]$ with

$$
G_{1}= \begin{cases}\frac{1}{r(1-c)} f\left(\frac{f(0)}{r(1-c)}\right) & \text { if }(\mathrm{H} 1) \text { holds } \\ \frac{f m}{r(1-c)} & \text { if }(\mathrm{H} 2)^{\prime} \text { holds }\end{cases}
$$

and

$$
G_{2}= \begin{cases}\frac{f(0)}{r(1-c)} & \text { if }(\mathrm{H} 1) \text { holds } \\ \frac{f_{M}}{r(1-c)} & \text { if }(\mathrm{H} 2)^{\prime} \text { holds }\end{cases}
$$

Then we know that from Lemma 3.1 all of the positive periodic solutions of Eq. (1) belong to the region $G$.

Lemma 3.2 Equation(1) has no nonconstant periodic solution when $\tau=0$.

Proof Obviously, when $\tau=0$, Eq. (1) becomes

$$
(1-c) \dot{N}=-r(1-c) N+f(N)
$$

Then the conclusion follows from the fact that the first order autonomous ODE has no nonconstant periodic solutions.

Remark 3.3 The proof of Lemma 3.2 is also able to show that Eq. (1) has no nonconstant periodic solution of period $\tau$.

\section{Lemma 3.4}

(i) If (H1) holds, then Eq. (1) has no nonconstant periodic solution of period $2 \tau$.

(ii) If $(\mathrm{H} 2)^{\prime}$ and $c f\left(G_{1}\right)<r\left(1-c^{2}\right) G_{1}$ hold, then Eq. (1) has no nonconstant periodic solution of period $2 \tau$.

Proof Let $u(t)$ be a nonconstant periodic solution to Eq. (1) of period $2 \tau$ and $v(t)=u(t-\tau)$. Then $u, v \in G$ and $(u(t), v(t))$ is a periodic solution to the following system of ordinary differential equations:

$$
\begin{aligned}
& \dot{u}-c \dot{v}=-r u+c r v+f(v), \\
& \dot{v}-c \dot{u}=-r v+c r u+f(u) .
\end{aligned}
$$


That is,

$$
\begin{aligned}
& \dot{u}=\frac{1}{1-c^{2}}\left(-r\left(1-c^{2}\right) u+c f(u)+f(v)\right):=P(u, v), \\
& \dot{v}=\frac{1}{1-c^{2}}\left(-r\left(1-c^{2}\right) v+f(u)+c f(v)\right):=Q(u, v) .
\end{aligned}
$$

Hence,

$$
\frac{\partial P}{\partial u}+\frac{\partial Q}{\partial v}=\frac{1}{1-c^{2}}\left(-2 r\left(1-c^{2}\right)+c\left(f^{\prime}(u)+f^{\prime}(v)\right)\right) .
$$

If (H1) holds, we have $f^{\prime}(s)<0$ for all $s \in G$. Thus, $\frac{\partial P}{\partial u}+\frac{\partial Q}{\partial v}<0$. If $(\mathrm{H} 2)^{\prime}$ holds, for all $s \in G$, we have

$$
f^{\prime}(s)<\frac{f(s)}{s} \leq \frac{f\left(G_{1}\right)}{G_{1}} .
$$

Then, from $c f\left(G_{1}\right)<r\left(1-c^{2}\right) G_{1}$, we also can conclude that $\frac{\partial P}{\partial u}+\frac{\partial Q}{\partial v}<0$. The classical Bendixson criterion implies that Eq. (20) has no nonconstant periodic solution in $G$. Therefore, Eq. (1) has no nonconstant periodic solution of period $2 \tau$. The proof is completed.

Denote

$$
R_{\min }=\min _{s \in G} f^{\prime}(s), \quad R_{\max }=\max _{s \in G} f^{\prime}(s), \quad \tilde{R}=\max \left\{\left|R_{\min }\right|,\left|R_{\max }\right|\right\}
$$

Then we have the following result.

Lemma 3.5 Assume that either $(\mathrm{H} 1)$ or $(\mathrm{H} 2)^{\prime}$ holds. If

$$
-2 r+2 r c^{4}+2 c^{3} R_{\max }+\left(\sqrt{2}+2 c+\sqrt{2} c^{2}\right) \tilde{R}<0
$$

then Eq. (1) has no nonconstant periodic solution of period $4 \tau$.

Proof Let $u(t)$ be a nonconstant periodic solution to Eq. (1) of period 4 and set $v_{i}(t)=u(t-$ $(i-1) \tau), i=1,2,3,4$. Then $V=\left(v_{1}(t), v_{2}(t), v_{3}(t), v_{4}(t)\right)$ is a nonconstant periodic solution to the following system of ordinary differential equations:

$$
\begin{aligned}
& \dot{v}_{1}-c \dot{v}_{2}=-r v_{1}+c r v_{2}+f\left(v_{2}\right), \\
& \dot{v}_{2}-c \dot{v}_{3}=-r v_{2}+c r v_{3}+f\left(v_{3}\right), \\
& \dot{v}_{3}-c \dot{v}_{4}=-r v_{3}+c r v_{4}+f\left(v_{4}\right), \\
& \dot{v}_{4}-c \dot{v}_{1}=-r v_{4}+c r v_{1}+f\left(v_{1}\right) .
\end{aligned}
$$


That is,

$$
\begin{aligned}
& \dot{v}_{1}=\frac{1}{1-c^{4}}\left(-\left(1-c^{4}\right) r v_{1}+c^{3} f\left(v_{1}\right)+f\left(v_{2}\right)+c f\left(v_{3}\right)+c^{2} f\left(v_{4}\right)\right), \\
& \dot{v}_{2}=\frac{1}{1-c^{4}}\left(-\left(1-c^{4}\right) r v_{2}+c^{2} f\left(v_{1}\right)+c^{3} f\left(v_{2}\right)+f\left(v_{3}\right)+c f\left(v_{4}\right)\right), \\
& \dot{v}_{3}=\frac{1}{1-c^{4}}\left(-\left(1-c^{4}\right) r v_{3}+c f\left(v_{1}\right)+c^{2} f\left(v_{2}\right)+c^{3} f\left(v_{3}\right)+f\left(v_{4}\right)\right), \\
& \dot{v}_{4}=\frac{1}{1-c^{4}}\left(-\left(1-c^{4}\right) r v_{4}+f\left(v_{1}\right)+c f\left(v_{2}\right)+c^{2} f\left(v_{3}\right)+c^{3} f\left(v_{4}\right)\right),
\end{aligned}
$$

whose orbits belong to $\tilde{G}:=\left\{V \in \mathbb{R}^{4} \mid v_{i} \in G, i=1,2,3,4\right\}$. Next, we will employ a general Bendixson criterion in higher dimensions developed in [32] to exclude nonconstant periodic solutions of Eq. (23) in region $G$, which will guarantee that there are no $4 \tau$-periodic solutions to Eq. (1). The Jacobian matrix $J(V)$ of Eq. (23), for $V \in \mathbb{R}^{4}$, is

$$
J(V)=-r I_{4 \times 4}+\frac{1}{1-c^{4}}\left(\begin{array}{cccc}
c^{3} f^{\prime}\left(v_{1}\right) & f^{\prime}\left(v_{2}\right) & c f^{\prime}\left(v_{3}\right) & c^{2} f^{\prime}\left(v_{4}\right) \\
c^{2} f^{\prime}\left(v_{1}\right) & c^{3} f^{\prime}\left(v_{2}\right) & f^{\prime}\left(v_{3}\right) & c f^{\prime}\left(v_{4}\right) \\
c f^{\prime}\left(v_{1}\right) & c^{2} f^{\prime}\left(v_{2}\right) & c^{3} f^{\prime}\left(v_{3}\right) & f^{\prime}\left(v_{4}\right) \\
f^{\prime}\left(v_{1}\right) & c f^{\prime}\left(v_{2}\right) & c^{2} f^{\prime}\left(v_{3}\right) & c^{3} f^{\prime}\left(v_{4}\right)
\end{array}\right)
$$

The second additive compound matrix $J^{[2]}(V)$ of $J(V)$ is

$$
J^{[2]}(V)=\frac{1}{1-c^{4}} \times S,
$$

where $S$ is a $6 \times 6$ matrix, whose rows $S_{i}$ are given in the following row vectors:

$$
\begin{aligned}
& S_{1}=\left(-2 r+2 r c^{4}+c^{3}\left(f^{\prime}\left(v_{1}\right)+f^{\prime}\left(v_{2}\right)\right), f^{\prime}\left(v_{3}\right), c f^{\prime}\left(v_{4}\right),-c f^{\prime}\left(v_{3}\right),-c^{2} f^{\prime}\left(v_{4}\right), 0\right), \\
& S_{2}=\left(c^{2} f^{\prime}\left(v_{2}\right),-2 r+2 r c^{4}+c^{3}\left(f^{\prime}\left(v_{1}\right)+f^{\prime}\left(v_{3}\right)\right), f^{\prime}\left(v_{4}\right), f^{\prime}\left(v_{2}\right), 0,-c^{2} f^{\prime}\left(v_{4}\right)\right), \\
& S_{3}=\left(c f^{\prime}\left(v_{2}\right), c^{2} f^{\prime}\left(v_{3}\right),-2 r+2 r c^{4}+c^{3}\left(f^{\prime}\left(v_{1}\right)+f^{\prime}\left(v_{4}\right)\right), 0, f^{\prime}\left(v_{2}\right), c f^{\prime}\left(v_{3}\right)\right), \\
& S_{4}=\left(-c f^{\prime}\left(v_{1}\right), c^{2} f^{\prime}\left(v_{1}\right), 0,-2 r+2 r c^{4}+c^{3}\left(f^{\prime}\left(v_{2}\right)+f^{\prime}\left(v_{3}\right)\right), f^{\prime}\left(v_{4}\right),-c f^{\prime}\left(v_{4}\right)\right), \\
& S_{5}=\left(-f^{\prime}\left(v_{1}\right), 0, c^{2} f^{\prime}\left(v_{1}\right), c^{2} f^{\prime}\left(v_{3}\right),-2 r+2 r c^{4}+c^{3}\left(f^{\prime}\left(v_{2}\right)+f^{\prime}\left(v_{4}\right)\right), f^{\prime}\left(v_{3}\right)\right), \\
& S_{6}=\left(0,-f^{\prime}\left(v_{1}\right), c f^{\prime}\left(v_{1}\right),-c f^{\prime}\left(v_{2}\right), c^{2} f^{\prime}\left(v_{2}\right),-2 r+2 r c^{4}+c^{3}\left(f^{\prime}\left(v_{3}\right)+f^{\prime}\left(v_{4}\right)\right)\right) .
\end{aligned}
$$

Choose $l_{\infty}$ norm in $\mathbb{R}^{6}$, namely, $|x|=\max _{1 \leq i \leq 6}\left|x_{i}\right|$. Let $A$ be the diagonal matrix given by

$$
A=\operatorname{diag}\{\sqrt{2}, 1, \sqrt{2}, \sqrt{2}, 1, \sqrt{2}\} .
$$

Then the Lozinskii measure of $A J^{[2]}(V) A^{-1}$ is

$$
\mu\left(A J^{[2]}(V) A^{-1}\right)=\frac{1}{1-c^{4}} \max \left\{\mu_{1}, \mu_{2}, \mu_{3}, \mu_{4}, \mu_{5}, \mu_{6}\right\}
$$


where

$$
\begin{aligned}
& \mu_{1}=-2 r+2 r c^{4}+c^{3}\left(f^{\prime}\left(v_{1}\right)+f^{\prime}\left(v_{2}\right)\right)+(\sqrt{2}+c)\left|f^{\prime}\left(v_{3}\right)\right|+\left(\sqrt{2} c^{2}+c\right)\left|f^{\prime}\left(v_{4}\right)\right|, \\
& \mu_{2}=-2 r+2 r c^{4}+c^{3}\left(f^{\prime}\left(v_{1}\right)+f^{\prime}\left(v_{3}\right)\right)+\frac{\sqrt{2}}{2}\left(1+c^{2}\right)\left(\left|f^{\prime}\left(v_{2}\right)\right|+\left|f^{\prime}\left(v_{4}\right)\right|\right), \\
& \mu_{3}=-2 r+2 r c^{4}+c^{3}\left(f^{\prime}\left(v_{1}\right)+f^{\prime}\left(v_{4}\right)\right)+(\sqrt{2}+c)\left|f^{\prime}\left(v_{2}\right)\right|+\left(\sqrt{2} c^{2}+c\right)\left|f^{\prime}\left(v_{3}\right)\right|, \\
& \mu_{4}=-2 r+2 r c^{4}+c^{3}\left(f^{\prime}\left(v_{2}\right)+f^{\prime}\left(v_{3}\right)\right)+(\sqrt{2}+c)\left|f^{\prime}\left(v_{4}\right)\right|+\left(\sqrt{2} c^{2}+c\right)\left|f^{\prime}\left(v_{1}\right)\right|, \\
& \mu_{5}=-2 r+2 r c^{4}+c^{3}\left(f^{\prime}\left(v_{2}\right)+f^{\prime}\left(v_{4}\right)\right)+\frac{\sqrt{2}}{2}\left(1+c^{2}\right)\left(\left|f^{\prime}\left(v_{1}\right)\right|+\left|f^{\prime}\left(v_{3}\right)\right|\right), \\
& \mu_{6}=-2 r+2 r c^{4}+c^{3}\left(f^{\prime}\left(v_{3}\right)+f^{\prime}\left(v_{4}\right)\right)+(\sqrt{2}+c)\left|f^{\prime}\left(v_{1}\right)\right|+\left(\sqrt{2} c^{2}+c\right)\left|f^{\prime}\left(v_{2}\right)\right| .
\end{aligned}
$$

Obviously, from Eq. (23), we have that $\mu\left(A J^{[2]}(V) A^{-1}\right)<0$ for $V \in \tilde{G}$, which is a Bendixson condition that rules out nonconstant periodic orbits of Eq. (23) in $\tilde{G}$ [32]. Therefore, Eq. (1) has no nonconstant periodic solution of period $4 \tau$. The proof is completed.

Now, we are in a position to state the following global Hopf bifurcation results.

Theorem 3.6 Assume that $r>0,0<c<1$, and $f$ is a nonnegative and $C^{2}$ function in $[0, \infty)$ with $f(\infty)=0$.

(i) If either $(\mathrm{H} 1)$ or $(\mathrm{H} 2)^{\prime}$ holds and $f^{\prime}\left(N_{0}\right)<-r(1+c)$ is satisfied, then Eq. (1) has at least one positive periodic solution for $\tau>\tau_{1}$.

(ii) If (H1), $-r\left(1+c^{2}\right)<c f^{\prime}\left(N_{0}\right)<-r c(1+c)$, and Eq. (22) hold, then Eq. (1) has at least one positive periodic solution for $\tau>\tau_{0}$ and two positive periodic solutions for $\tau>\tau_{1}$.

(iii) If $(\mathrm{H} 2)^{\prime},-r\left(1+c^{2}\right)<c f^{\prime}\left(N_{0}\right)<-r c(1+c), c f\left(G_{1}\right)<r\left(1-c^{2}\right) G_{1}$, and Eq. (22) hold, then Eq. (1) has at least one positive periodic solution for $\tau>\tau_{0}$ and two positive periodic solutions for $\tau>\tau_{1}$.

Proof First, from the definition of $\tau_{j}$ in Eq. (9), we know that $\tau_{j}<\tau_{j+1}$. Define

$$
\begin{aligned}
& X=C([-\tau, 0], \mathbb{R}), \\
& \Sigma=C l\{(y, \tau, T): y \text { is a } T \text {-periodic solution of Eq. (1) }\} \subset X \times \mathbb{R}_{+} \times \mathbb{R}_{+},
\end{aligned}
$$

and denote by $C\left(N_{0}, \tau_{j}, \frac{2 \pi}{\omega_{0}}\right)$ the connected component of $\left(N_{0}, \tau_{j}, \frac{2 \pi}{\omega_{0}}\right)$ in $\Sigma$, where $\omega_{0}$ is defined in Eq. (8). Note that, under assumption (H1) or $(\mathrm{H} 2)^{\prime}$, the stationary points $\left(N_{0}, \tau_{j}, \frac{2 \pi}{\omega_{0}}\right)$ of Eq. (1) are nonsingular and they are isolated centers (see [31]). Thus, Lemma 2.6 implies that there exist $\epsilon>0, \delta>0$, and a smooth curve $\lambda:\left(\tau_{j}-\delta, \tau_{j}+\delta\right) \rightarrow \mathbb{C}$ such that

$$
\Delta(\lambda(\tau))=\Delta_{\left(N_{0}, \tau, T\right)}(\lambda(\tau))=0, \quad\left|\lambda(\tau)-i \omega_{0}\right|<\epsilon,
$$

for all $\tau \in\left[\tau_{j}-\delta, \tau_{j}+\delta\right]$, where $\Delta$ is defined as (6), and

$$
\lambda\left(\tau_{j}\right)=i \omega_{0},\left.\quad \frac{d \operatorname{Re}(\lambda(\tau))}{d \tau}\right|_{\tau=\tau_{j}}>0
$$


Let

$$
\Omega_{\epsilon}=\left\{(u, p): 0<u<\epsilon,\left|p-\frac{2 \pi}{\omega_{0}}\right|<\epsilon\right\} .
$$

It is easy to see that on $\left[\tau_{j}-\delta, \tau_{j}+\delta\right] \times \bar{\Omega}_{\epsilon}, \Delta_{\left(N_{0}, \tau, T\right)}\left(u+\frac{2 \pi i}{p}\right)=0$ if and only if $\tau=\tau_{j}, u=0$, and $p=\frac{2 \pi}{\omega_{0}}$. Moreover, put

$$
H^{ \pm}\left(N_{0}, \tau_{j}, \frac{2 \pi}{\omega_{0}}\right)(u, p)=\Delta_{\left(N_{0}, \tau \pm \delta, T\right)}\left(u+\frac{2 \pi i}{p}\right) .
$$

Then, for any $j=0,1,2, \ldots$, the crossing number is

$$
\begin{aligned}
\gamma_{1}\left(N_{0}, \tau_{j}, \frac{2 \pi}{\omega_{0}}\right) & =\operatorname{deg}_{B}\left(H^{-}\left(N_{0}, \tau_{j}, \frac{2 \pi}{\omega_{0}}\right), \Omega_{\epsilon}\right)-\operatorname{deg}_{B}\left(H^{+}\left(N_{0}, \tau_{j}, \frac{2 \pi}{\omega_{0}}\right), \Omega_{\epsilon}\right) \\
& =-1 .
\end{aligned}
$$

It follows that

$$
\Sigma_{\left(N_{0}, \tau, T\right) \in C\left(N_{0}, \tau_{j}, \frac{2 \pi}{\omega_{0}}\right)} \gamma_{1}\left(N_{0}, \tau, T\right)<0 .
$$

Therefore, we conclude that the connected component is nonempty and unbounded from the local and global Hopf bifurcation theorem given by Krawcewicz, Wu, and Xia (see [31]).

By Lemma 3.1, the projection of $C\left(N_{0}, \tau_{j}, \frac{2 \pi}{\omega_{0}}\right)$ onto the $y$-space is bounded. Meanwhile, the projection of $C\left(N_{0}, \tau_{j}, \frac{2 \pi}{\omega_{0}}\right)$ onto $\tau$-space is bounded below from Lemma 3.2.

From Eq. (9), we know that

$$
2 j \pi<\omega_{0} \tau_{j}<2(j+1) \pi, \quad j \geq 1,
$$

which implies

$$
\frac{1}{j+1}<\frac{2 \pi}{\omega_{0} \tau_{j}}<\frac{1}{j}, \quad j \geq 1
$$

By Remark 3.3, for $j \geq 1$, one has that $\frac{\tau}{j+1}<T<\frac{\tau}{j}$ if $(y, \tau, T) \in C\left(N_{0}, \tau_{j}, \frac{2 \pi}{\omega_{0}}\right)$. This fact shows that the projection of $C\left(N_{0}, \tau_{j}, \frac{2 \pi}{\omega_{0}}\right)$ onto the $T$-space is bounded if $\tau$ is bounded. Thus, in order for $C\left(N_{0}, \tau_{j}, \frac{2 \pi}{\omega_{0}}\right)$ to be unbounded, its projection onto the $\tau$-space must be unbounded. In other words, the projection of $C\left(N_{0}, \tau_{j}, \frac{2 \pi}{\omega_{0}}\right)$ onto the $\tau$-space includes $\left[\tau_{j}, \infty\right)$. The proof of (i) is completed.

If either $(\mathrm{H} 1)$ or $(\mathrm{H} 2)^{\prime}$ holds and $-r\left(1+c^{2}\right)<c f^{\prime}\left(N_{0}\right)<-r c(1+c)$ is satisfied, then from Eq. (7), Eq. (8), and Eq. (9), we have

$$
\begin{aligned}
\cos \omega_{0} \tau_{0} & =\frac{r\left(c r+f^{\prime}\left(N_{0}\right)\right)+c \omega_{0}^{2}}{r^{2}+\omega_{0}^{2}} \\
& =\frac{f^{\prime}\left(N_{0}\right)\left(r\left(1+c^{2}\right)+c f^{\prime}\left(N_{0}\right)\right)}{r^{2}+\omega_{0}^{2}}<0,
\end{aligned}
$$


which implies

$$
2<\frac{2 \pi}{\omega_{0} \tau_{0}}<4
$$

By Lemmas 3.4 and 3.5, if (H1) and Eq. (22) hold, we have $2 \tau<T<4 \tau$ when $(y, \tau, T) \in$ $C\left(N_{0}, \tau_{0}, \frac{2 \pi}{\omega_{0}}\right)$. It follows that the projection of $C\left(N_{0}, \tau_{0}, \frac{2 \pi}{\omega_{0}}\right)$ onto the $T$-space is bounded if $\tau$ is bounded. Thus, the projection onto $\tau$-space of $C\left(N_{0}, \tau_{0}, \frac{2 \pi}{\omega_{0}}\right)$ must be unbounded. In other words, the projection of $C\left(N_{0}, \tau_{j}, \frac{2 \pi}{\omega_{0}}\right)$ onto the $\tau$-space includes $\left[\tau_{0}, \infty\right)$. The proof of (ii) is completed.

Similarly, if (H2)', $c f\left(G_{1}\right)<r\left(1-c^{2}\right) G_{1}$ and Eq. (22) hold, we also have $2 \tau<T<4 \tau$ when $(y, \tau, T) \in C\left(N_{0}, \tau_{0}, \frac{2 \pi}{\omega_{0}}\right)$. Therefore, the projection onto the $\tau$-space of $C\left(N_{0}, \tau_{0}, \frac{2 \pi}{\omega_{0}}\right)$ must include $\left[\tau_{0}, \infty\right)$. The proof of (iii) is completed.

\section{Two examples and simulations}

In this section, we carry out some numerical simulations for Eq. (1).

\subsection{Example 1: neutral Nicholson's blowflies model}

Choose $f(s)=p s e^{-\alpha s}$ and consider the following neutral Nicholson's blowflies model:

$$
\dot{N}(t)-c \dot{N}(t-\tau)=-r N(t)+c r N(t-\tau)+p N(t-\tau) e^{-\alpha N(t-\tau)} .
$$

We would like to mention that the authors of reference [30] have investigated the existence of global Hopf bifurcation of Eq. (24). However, the global Hopf bifurcation conditions given in [30] depend on the existence of a positive real number, which is denoted by $\beta$ in [30], and are very difficult to verify. In this subsection, we take the parameter values as follows:

$$
r=0.5, \quad c=0.1, \quad p=4.5, \quad \alpha=3,
$$

and show that this set of parameters satisfies the global Hopf bifurcation conditions in this paper.

Firstly, we have that $f^{\prime}(0)=p>r(1-c)$. Thus, (H2) holds and Eq. (24) has a unique positive equilibrium $N_{0}=0.7675$. By calculation, we have $f^{\prime}\left(N_{0}\right)=-0.5862<-r(1+c)=$ -0.55 . Then, from Theorem 2.7 (ii), $N=N_{0}$ is locally asymptotically stable for $\tau \in\left[0, \tau_{0}\right)$ (see Fig. 1(a)) and unstable for $\tau>\tau_{0}$, and system (24) undergoes a local Hopf bifurcation at $N=N_{0}$ when $\tau=\tau_{j}, j=0,1,2, \ldots$, where

$$
\tau_{0}=14.0542, \quad \tau_{1}=46.3503, \quad \tau_{2}=78.6463,
$$

Next, we check the conditions of the global Hopf bifurcation (Theorem 3.6(iii)). Obviously, $-r\left(1+c^{2}\right)<c f^{\prime}\left(N_{0}\right)$ is satisfied. From the definitions given by Eq. (14), Eq. (17), 


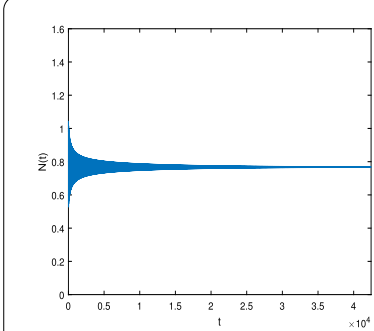

(a) $\tau=13.5$

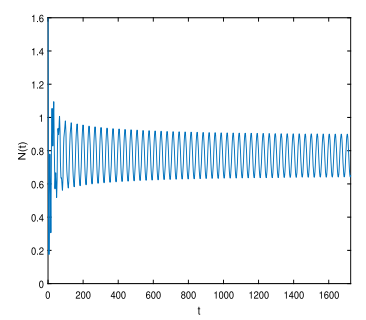

(b) $\tau=14.5$

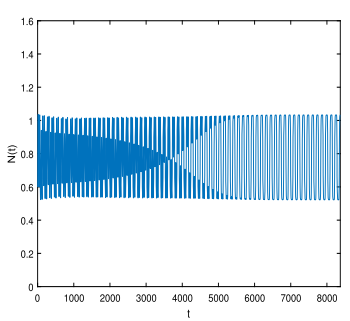

(c) $\tau=62$

Figure 1 (a) A solution converges to the positive equilibrium $N_{0}$ for $\tau=13.5<\tau_{0}=14.0542$. (b) A solution converges to a stable periodic solution for $\tau=14.5>\tau_{0}$. (c) A solution first approaches an unstable periodic solution before it converges to a stable periodic solution for $\tau=62>\tau_{1}=46.3503$

Eq. (18), Eq. (19), and Eq. (21), we have

$$
\begin{aligned}
& f_{M}=f\left(\frac{1}{a}\right)=0.5518, \quad f_{m}=f\left(\frac{f_{M}}{r(1-c)}\right)=0.1394, \\
& G_{1}=\frac{f_{m}}{r(1-c)}=0.3097, \quad G_{2}=\frac{f_{M}}{r(1-c)}=1.2263, \\
& R_{\min }=f^{\prime}\left(\frac{2}{a}\right)=-0.6090, \quad R_{\max }=f^{\prime}\left(G_{1}\right)=0.1261, \\
& \tilde{R}=\left|R_{\min }\right|=0.6090 .
\end{aligned}
$$

It follows that

$$
c f\left(G_{1}\right)=0.0550<r\left(1-c^{2}\right) G_{1}=0.1533
$$

and

$$
-2 r+2 r c^{4}+2 c^{3} R_{\max }+\left(\sqrt{2}+2 c+\sqrt{2} c^{2}\right) \tilde{R}=-0.0080<0 .
$$

Therefore, the conditions of Theorem 3.6(iii) are all satisfied, and Eq. (24) has at least one periodic solution for $\tau>\tau_{0}$ (see Fig. 1(b)) and two periodic solutions for $\tau>\tau_{1}$ (see Fig. 1(c)).

\subsection{Example 2: neutral Mackey-Glass model}

Choose $f(s)=\frac{b s}{1+s^{3}}$ and consider the following neutral Mackey-Glass model:

$$
\dot{N}(t)-c \dot{N}(t-\tau)=-r N(t)+c r N(t-\tau)+\frac{b N(t-\tau)}{1+N^{3}(t-\tau)},
$$

with the parameters $r=0.5, c=0.1, b=1.8$.

Obviously, we have that $f^{\prime}(0)=b>r(1-c)$. It follows that (H2)' holds and Eq. (25) has a unique positive equilibrium $N_{0}=1.4422$. Moreover, we can obtain that $f^{\prime}\left(N_{0}\right)=-0.5625<$ $-r(1+c)=-0.55$. Then, from Theorem 2.7 (ii), $N=N_{0}$ is locally asymptotically stable for $\tau \in\left[0, \tau_{0}\right)$ (see Fig. 2(a)) and unstable for $\tau>\tau_{0}$, and system (25) undergoes a local Hopf 


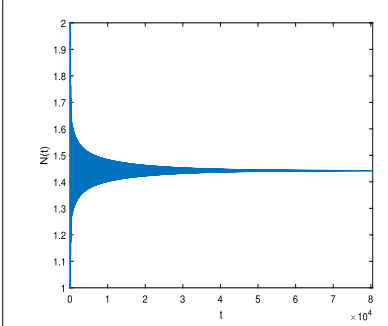

(a) $\tau=24.5$

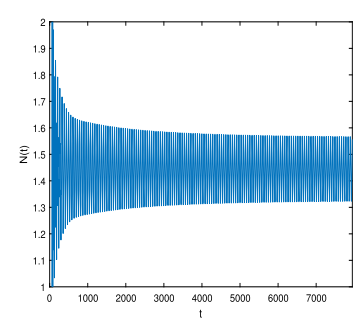

(b) $\tau=26.5$

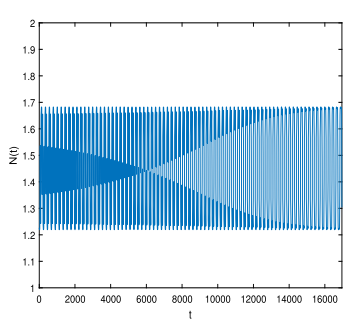

(c) $\tau=108$

Figure 2 (a) A solution converges to the positive equilibrium $N_{0}$ for $\tau=24.5<\tau_{0}=25.6233$. (b) A solution converges to a stable periodic solution for $\tau=26.5>\tau_{0}$. (c) A solution first approaches an unstable periodic solution before it converges to a stable periodic solution for $\tau=108>\tau_{1}=81.1939$

bifurcation at $N=N_{0}$ when $\tau=\tau_{j}, j=0,1,2, \ldots$, where

$$
\tau_{0}=25.6233, \quad \tau_{1}=81.1939, \quad \tau_{2}=136.7644,
$$

Now, we check the conditions of the global Hopf bifurcation (Theorem 3.6(iii)). Similarly, we can obtain that $-r\left(1+c^{2}\right)<c f^{\prime}\left(N_{0}\right)$ and

$$
\begin{aligned}
& f_{M}=f\left(2^{-\frac{1}{3}}\right)=0.9524, \quad f_{m}=f\left(\frac{f_{M}}{r(1-c)}\right)=0.3635, \\
& G_{1}=\frac{f_{m}}{r(1-c)}=0.8077, \quad G_{2}=\frac{f_{M}}{r(1-c)}=2.1165, \\
& R_{\min }=f^{\prime}\left(2^{\frac{1}{3}}\right)=-0.6000, \quad R_{\max }=f^{\prime}\left(G_{1}\right)=0.0416, \\
& \tilde{R}=\left|R_{\min }\right|=0.6000 .
\end{aligned}
$$

It follows that

$$
c f\left(G_{1}\right)=0.0952<r\left(1-c^{2}\right) G_{1}=0.3998
$$

and

$$
-2 r+2 r c^{4}+2 c^{3} R_{\max }+\left(\sqrt{2}+2 c+\sqrt{2} c^{2}\right) \tilde{R}=-0.0230<0 .
$$

Therefore, the conditions of Theorem 3.6(iii) are all satisfied, and Eq. (25) has at least one periodic solution for $\tau>\tau_{0}$ (see Fig. 2(b)) and two periodic solutions for $\tau>\tau_{1}$ (see Fig. 2(c)).

\section{Acknowledgements}

The authors are very grateful to the referees for their valuable suggestions.

\section{Funding}

This work is supported by the Fundamental Research Funds for the Central Universities (No. 2572020BC09).

Availability of data and materials

Not applicable.

Ethics approval and consent to participate

Not applicable. 


\section{Competing interests}

The authors declare that they have no competing interests.

\section{Consent for publication}

Not applicable.

\section{Authors' contributions}

All authors participated in the writing and coordination of the manuscript and all authors read and approved the final manuscript.

\section{Author details}

${ }^{1}$ Northeast Forestry University, Harbin, Heilongjiang 150040, P.R. China. ${ }^{2}$ Heilongjiang University, Harbin, Heilongjiang 150080, P.R. China.

\section{Publisher's Note}

Springer Nature remains neutral with regard to jurisdictional claims in published maps and institutional affiliations.

Received: 19 September 2020 Accepted: 11 February 2021 Published online: 17 May 2021

\section{References}

1. Bocharov, G., Hadeler, K.: Structured population models, conservation laws, and delay equations. J. Differ. Equ. 168, 212-237 (2000)

2. Kulenovic, M., Ladas, G., Sficas, Y.: Global attractivity in Nicholson's blowflies. Appl. Anal. 43, 109-124 (1992)

3. Feng, Q., Yan, J.: Global attractivity and oscillation in a kind of Nicholson's blowflies. J. Biomath. 17, 21-26 (2002)

4. Györi, I., Trofimchuk, S.: On the existence of rapidly oscillatory solutions in the Nicholson's blowflies equation. Nonlinear Anal. TMA 48, 1033-1042 (2002)

5. Li, J.: Global attractivity in Nicholson's blowflies. Appl. Math. Ser. B 11, 425-434 (1996)

6. Wei, J., Li, M.: Hopf bifurcation analysis in a delayed Nicholson's blowflies equation. Nonlinear Anal. TMA 60, 1351-1367 (2005)

7. Sakar, S., Agarwal, S.: Oscillation and global attractivity in a periodic Nicholson's blowflies model. Math. Comput. Model. 35, 719-731 (2002)

8. Weng, P., Liang, M.: Existence and global attractivity of periodic solution of a model in population dynamics. Acta Math. Appl. Sin. Engl. Ser. 12, 427-434 (1996)

9. So, J., Yu, J.: On the stability and uniform persistence of a discrete model of Nicholson's blowflies. J. Math. Anal. Appl. 193, 233-244 (1995)

10. Kocić, V., Ladas, G.: Oscillation and global attractivity in a discrete model of Nicholson's blowflies. Appl. Anal. 38 21-31 (1990)

11. Karakostas, G., Philos, C., Sficas, Y.: The dynamics of some discrete population models. Nonlinear Anal. TMA 17 1069-1084 (1991)

12. Zhang, B., Xu, H.: A note on the global attractivity of a discrete model of Nicholson's blowflies. Discrete Dyn. Nat. Soc. 3, 51-55 (1999)

13. So, J.W.-H., Zou, X.F.: Traveling waves for the diffusive Nicholson's blowflies equation. Appl. Math. Comput. 122, 385-392 (2001)

14. Yi, T.S., Zou, X.F.: Global attractivity of the diffusive Nicholson blowflies equation with Neumann boundary condition: a non-monotone case. J. Differ. Equ. 245, 3376-3388 (2008)

15. So, J.W.-H., Yang, Y.: Dirichlet problem for the diffusive Nicholson's blowflies equation. J. Differ. Equ. 150, 317-348 (1998)

16. Su, Y., Wei, J.J., Shi, J.P.: Bifurcation analysis in a delayed diffusive Nicholson's blowflies equation. Nonlinear Anal., Real World Appl. 11, 1692-1703 (2010)

17. Pang, P., Wang, Y.: Time periodic solutions of the diffusive Nicholson blowflies equation with delay. Nonlinear Anal., Real World Appl. 22, 44-53 (2015)

18. Karakostas, G., Philos, C., Sficas, Y.: Stable steady state of some population models. J. Dyn. Differ. Equ. 4(1), 161-190 (1992)

19. Saker, S.: Oscillation and global attractivity in hematopoiesis model with delay time. Appl. Math. Comput. 136, 241-250 (2003)

20. Wan, A., Wei, J.: Bifurcation analysis of Mackey-Glass electronic circuits model with delayed feedback. Nonlinear Dyn. 57(1-2), 85-96 (2009)

21. Gopalsamy, K., Kulenovic, M., Ladas, G.: Oscillations and global attractivity in haematopoiesis. J. Dyn. Differ. Equ. 2 117-132 (1990)

22. Song, Y., Wei, J.: Bifurcation analysis on a survival red blood cells model. J. Math. Anal. Appl. 316, 458-471 (2006)

23. Fan, D., Wei, J.: Hopf bifurcation analysis in a Mackey-Glass system. Int. J. Bifurc. Chaos 17, 2149-2157 (2007)

24. Mackey, M.C., Glass, L.: Oscillation and chaos in physiological control system. Science 197, $287-289$ (1977)

25. Namajūnas, A., Pyragas, K., Tamaševičius, A.: An electronic analog of the Mackey-Glass system. Phys. Lett. A 201, 42-46 (1995)

26. Losson, J., Mackey, M.C., Longtin, A.: Solution multistability in first-order nonlinear differential delay equations. Chaos 3, 167-176 (1993)

27. Li, T., Pintus, N., Viglialoro, G.: Properties of solutions to porous medium problems with different sources and boundary conditions. Z. Angew. Math. Phys. 70(3), Art. 86, 1-18 (2019)

28. Viglialoro, G.: Very weak global solutions to a parabolic-parabolic chemotaxis-system with logistic source. J. Math Anal. Appl. 439(1), 197-212 (2016)

29. Wei, J.: Bifurcation analysis in a scalar delay differential equation. Nonlinearity 20, 2483-2498 (2007) 
30. Li, M., Wang, C., Wei, J.: Global Hopf bifurcation analysis of a Nicholson's blowflies equation of neutral type. J. Dyn. Differ. Equ. 26, 165-179 (2014)

31. Krawcewicz, W., Wu, J., Xia, H.: Global Hopf bifurcation theory for condensing fields and neutral equations with applications to lossless transmission problems. Can. Appl. Math. Q. 1, 167-219 (1993)

32. Li, M., Muldowney, J.: On Bendixson's criterion. J. Differ. Equ. 106, 27-39 (1993)

Submit your manuscript to a SpringerOpen ${ }^{\odot}$ journal and benefit from:

- Convenient online submission

- Rigorous peer review

- Open access: articles freely available online

- High visibility within the field

- Retaining the copyright to your article

Submit your next manuscript at $\boldsymbol{~ s p r i n g e r o p e n . c o m ~}$ 